\title{
Cardiac PET reimbursement policy: Success when we come together
}

\author{
Randall C. Thompson, MD, FASNC ${ }^{a}$ \\ a St. Luke's Mid America Heart Institute, Kansas City, MO
}

Received Feb 6, 2020; accepted Feb 7, 2020

doi: $10.1007 / \mathrm{s} 12350-020-02077-5$

In recent months, scores of volunteers and staff from ASNC and other medical societies have spent countless hours crafting new billing codes, establishing appropriate valuations, and educating policymakers on the importance of cardiac PET imaging services for medical care. There has been absolutely superb cooperation and coordination among medical societies and between various stake holders. It appears that these efforts have generally been successful, at least for now, and that crucial cardiac PET services will continue to be available to our patients. The recent work is a model of how appropriate policy can be shaped by an engaged medical community.

\section{WHAT IS NEW}

Beginning January 1, 2020, there are several important changes in the AMA's Current Procedural Terminology $\left(\mathrm{CPT}^{\mathrm{TM}}\right)$ billing codes for cardiac PET. There are also changes in the billing codes for nuclear medicine SPECT. The latter are relevant to cardiac imagers because of the codes used to report amyloid imaging with SPECT (e.g., CPT codes 78803, 78830). Both families of CPT Codes have been updated to reflect changes in technology, and there are new codes for use of imaging with hybrid CT machines. Cardiac SPECT codes, on the other hand, are not affected. The new codes better capture the cost of the advanced instrumentation and the extra work physicians provide in reviewing both the emission images and the CT attenuation map. Also, for cardiac PET, the code for absolute quantitation of myocardial blood flow was upgraded from a temporary Category III code (0482T) to a permanent Category I code (78434). There are also two

Reprint requests: Randall C. Thompson, MD, FASNC, St. Luke's Mid America Heart Institute, 4330 Wornall Rd, Kansas City, MO 64111; rthompson@saint-lukes.org

J Nucl Cardiol 2020;27:345-7.

$1071-3581 / \$ 34.00$

Copyright (c) 2020 American Society of Nuclear Cardiology. new codes for dual radiotracer cardiac PET for metabolism and perfusion (78432, 78433) (see Table 1). These codes are used to report myocardial viability imaging and to report imaging for cardiac sarcoid. As an aside, PET cardiac sarcoid imaging is not currently approved for reimbursement in the USA by The Centers for Medicare and Medicaid Services (CMS). PET infection/inflammation imaging is subject to a negative CMS National Coverage Determination (NCD) dating back to 2008, although ASNC and other societies are filing a petition to retire that NCD. If successful, local Medicare carriers would be free to set their own policy and reimbursement standards for PET sarcoid and infection imaging.

\section{THE CMS PROPOSED RULE FOR CARDIAC PET}

In summer 2019, CMS issued their proposed rule for reimbursement for the new cardiac PET codes for CY 2020. ASNC, the American College of Cardiology (ACC), and the Society of Nuclear Medicine and Molecular Imaging (SNMMI) identified several suspected errors in the calculation of the reimbursement rates and provided extensive comments. Several delegations from the societies visited with representative from CMS, additional cost input was provided and numerous individual physicians wrote to comment. In addition to the medical societies, industry partners visited CMS and provided further data. The feedback provided by ASNC and others during this official comment period was largely successful. It is very clear that the appropriate personnel at CMS heard the concerns of our community and were led to understand the important role of PET cardiac imaging for modern patient care. For example, the 2019 final rule states: "In the interest of maintaining payment stability and protecting patient access to these important services,...". ${ }^{1}$ The dedicated professionals at CMS engaged with stakeholders and allowed the dialog that helped to work through complex issues. 
Table 1. Calendar year 2020 cardiac PET current procedural terminology codes

\section{CPT \\ code}

\section{Description}

78459 Myocardial imaging, positron emission tomography (PET), metabolic evaluation study (including ventricular wall motion(s), and/or ejection fraction(s), when performed) single study

78429 Myocardial imaging, positron emission tomography (PET), metabolic evaluation study (including ventricular wall motion(s), and/or ejection fraction(s), when performed) single study; with concurrently acquired computed tomography transmission scan

78491 Myocardial imaging, positron emission tomography, perfusion study (including ventricular wall motion(s), and/or ejection fractions(s), when performed); single study, at rest or stress (exercise or pharmacologic)

78430 Myocardial imaging, positron emission tomography, perfusion study (including ventricular wall motion(s), and/or ejection fractions(s), when performed); single study, at rest or stress (exercise or pharmacologic), with concurrently acquired computed tomography transmission scan

78492 Myocardial imaging, positron emission tomography, perfusion study (including ventricular wall motion(s), and/or ejection fractions(s), when performed); multiple studies at rest and stress (exercise or pharmacologic)

78431 Myocardial imaging, positron emission tomography, perfusion study (including ventricular wall motion(s), and/or ejection fractions(s), when performed); multiple studies at rest and stress (exercise or pharmacologic), with concurrently acquired computed tomography transmission scan

+78434 Absolute quantitation of myocardial blood flow (AQMBF), positron emission tomography, rest and pharmacologic stress (List separately in addition to code for primary procedure)

78432 Myocardial imaging, positron emission tomography, combined perfusion with metabolic evaluation study (including ventricular wall motion(s), and/or ejection fraction(s), when performed), dual radiotracer (eg, myocardial viability)

78433 Myocardial imaging, positron emission tomography, combined perfusion with metabolic evaluation study (including ventricular wall motion(s), and/or ejection fraction(s), when performed), dual radiotracer (eg, myocardial viability); with concurrently acquired computed tomography transmission scan

\section{PHYSICIAN FEE SCHEDULE VS HOSPITAL OUTPATIENT PAYMENT SYSTEM}

In the USA, services such as cardiac PET imaging are billed under either the Physician Fee Schedule (PFS) (freestanding imaging centers or physician offices) or The Hospital Outpatient Perspective Payment System (HOPPS) for hospital outpatient imaging. By statute, the calculation of the CMS fee schedule for the technical component of the service is different for these two pathways. The physician work relative value unit (RVU) is the same for HOPPS and PFS, however. The house of medicine, working through the AMA's Relative Value Update Committee (RUC) had proposed a set of work RVUs for the new cardiac PET codes. In the draft CMS rule, the work RVUs would have been cut significantly from the RUC proposed valuation. After the comment period and considerable feedback from ASNC and other stakeholders, CMS agreed that the RUC valued work RVU's were appropriate, and reinstated them.

\section{PHYSICIAN FEE SERVICE TECHNICAL VALUE}

CMS also proposed what would have amounted to sharp cuts in the technical reimbursement for cardiac PET under the PFS. Up until now, cardiac PET PFS has been set by individual Medicare carriers. Policy makers at CMS did not have those values to review. ASNC and other stakeholders also pointed out several errors in the inputs used for the new PET PFS calculations. For example, the cost of PET scanners were undervalued and the assumed utilization rates were much higher than would be typical for equipment of this category. Again, the policymakers at CMS heard the concerns of our community and agreed that such severe price instability would be detrimental. For 2020, cardiac PET services under PFS will remain carrier priced. ASNC and other stakeholders have worked diligently to provide the additional complex cost information that CMS needed for accurate calculations, meeting the February 10 deadline. PET technical reimbursement rates under 
PFS for future years have not yet been determined, but now policymakers have the complicated data input needed for their calculations.

\section{HOSPITAL OUTPATIENT NEW TECHNOLOGY AMBULATORY PAYMENT CATEGORY}

As mentioned above, the calculation of the technical part of the cost of a service are statutorily different under PFS and HOPPS. With HOPPS, services such as cardiac PET imaging are grouped under Ambulatory Payment Categories (APCs), analogous to inpatient Diagnosis-related Groups (DRG's). Similar services are grouped and almost all individual components of performing the service are included. ASNC and SNMMI pointed out to policymakers at CMS that three of the new PET CPT codes involved considerably greater technical complexity and cost than would be covered by the then current APC value. The cost of the PET-CT instrumentation is greater than for a dedicated PET-CT machine used in prior calculations, the cost of the dual radiotracer service involves the price of two, rather than one radiotracer, and there should be some allowance for the technical cost of providing absolute quantitation of myocardial blood flow. CMS agreed, and for calendar years 2020, CPT codes 78430, 78432, and 78433 have been placed in a New Technology APC which allows for somewhat higher reimbursement. One point of occasional confusion on the part of hospital billing personnel is that absolute quantitation of myocardial blood flow (78434) is an add-on code for the physician work RVU, but the technical component, while reported, the payment is included in the APC.

\section{WHAT WAS EFFECTIVE}

Large numbers of volunteers and staff from ASNC and other medical societies worked countless hours on the process that involves describing new CPT codes, valuing those codes, and interacting with CMS and other payers in order to get proper reimbursement. This system is complicated and the effort by our community on behalf of our patients has been herculean. There is still work to do, but in recent months the tremendous cooperation and coordination among medical societies and between various stake holders is a model of what can be accomplished by organized medicine and engaged members. It appears that our community's efforts have generally been successful, and that important cardiac PET services will continue to be available to our patients.

\section{Disclosure}

The author Randall C. Thompson reports no conflicts of interest.

\section{References}

1. Medicare Program. CY 2020 revisions to payment policies under the physician fee schedule... https://www.federalregister.gov/docu ments/2019/11/15/2019-24086/medicare-program-cy-2020-revision s-to-payment-policies-under-the-physician-fee-schedule-and-other. Accessed 4 Feb 2020.

Publisher's Note Springer Nature remains neutral with regard to jurisdictional claims in published maps and institutional affiliations. 